\section{Intersections}

Canadian Journal of Music

Revue canadienne de musique
Intersections CANADIAN JOURAL OF MUSIC
REVUE CANADIENEE DE MUSIOUH

\title{
Quand le metal flirte avec ABBA : Analyse des transformations formelles et thématiques de "Summer Night City "
}

\section{Méi-Ra St-Laurent}

Volume 34, numéro 1-2, 2014

URI : https://id.erudit.org/iderudit/1030873ar

DOI : https://doi.org/10.7202/1030873ar

Aller au sommaire du numéro

Éditeur(s)

Canadian University Music Society / Société de musique des universités canadiennes

ISSN

1911-0146 (imprimé)

1918-512X (numérique)

Découvrir la revue

Citer cet article

St-Laurent, M.-R. (2014). Quand le metal flirte avec ABBA : Analyse des transformations formelles et thématiques de "Summer Night City ". Intersections, 34(1-2), 135-151. https://doi.org/10.7202/1030873ar
Résumé de l'article

Une des caractéristiques fondamentales de la musique populaire demeure la réutilisation constante du matériel musical en provenance d'une multitude de styles et de courants (remix, adaptation, échantillonnage, etc.). Cette pratique nommée la transphonographie provient de l'intertextualité, un concept élaboré par le littéraire Gérard Genette et adapté à la musique populaire par le musicologue Serge Lacasse. Dans ce texte, l'auteur emploiera la transphonographie pour relever les différentes transformations musicales et thématiques survenant dans la chanson « Summer Night City » de ABBA, lorsque celle-ci est reprise par le groupe de gothique metal symphonique Therion. En plus des éléments musicaux propres à la musique d'ABBA modifiés pour correspondre à l'esthétique de Therion, ces transformations induisent aussi un changement dans la signification même de la chanson.
Copyright (C Canadian University Music Society / Société de musique des universités canadiennes, 2015
Ce document est protégé par la loi sur le droit d'auteur. L’utilisation des services d’Érudit (y compris la reproduction) est assujettie à sa politique d'utilisation que vous pouvez consulter en ligne.

https://apropos.erudit.org/fr/usagers/politique-dutilisation/ 


\title{
QUAND LE METAL FLIRTE AVEC ABBA : ANALYSE DES TRANSFORMATIONS FORMELLES ET THÉMATIQUES DE «SUMMER NIGHT CITY»
}

\author{
Méi-Ra St-Laurent
}

Depuis le début de sa carrière en 1972, le groupe culte suédois ABBA a été une source d'inspiration majeure pour différents musiciens, tant electrodance, funk ${ }^{1}$ que metal. Ainsi, les différentes reprises des chansons de ABBA pourraient être qualifiées de «transtylisation», puisque les musiciens ne se contentent pas seulement de réutiliser la musique de ABBA, ils l'adaptent à leur propre style. Au-delà des transtylisations qu'une telle pratique peut entraîner (p. ex. adaptation de la mélodie, modification des inflexions vocales, ajout d'effets sonores), plusieurs transformations d'ordre thématique peuvent également survenir (p. ex. modification du récit ou de la signification de la chanson). Ainsi en 2001, Therion, un groupe suédois de gothique metal symphonique, exécute ce qu'il conviendrait d'appeler une "transposition" (j'y reviendrai) de la chanson et du vidéoclip «Summer Night City» de ABBA². En effet, il exécute non seulement des transformations formelles ${ }^{3}$ de la chanson ABBA en modifiant la structure musicale (transtylisation) pour qu'elle corresponde à l'esthétique metal symphonique, mais en adapte aussi la thématique pour qu'elle s'inscrive davantage dans l'idéologie metal. Ce choc esthétique soulève donc la question suivante : quelles sont les transformations formelles et thématiques présentes dans la version de Therion et comment influencent-elles notre regard sur l'hypertexte?

Dans cette analyse, je relèverai d'abord les différentes transformations formelles (influençant la structure musicale) présentes dans la transposition de Therion en me référant à l'enregistrement sonore. Je m'attarderai ensuite aux principales transformations thématiques présentes dans le vidéoclip de la chanson "Summer Night City», en m’intéressant aux dimensions diégétiques, narratives, pragmatiques et sémantiques. Sur le plan méthodologique, je baserai mon analyse sur la notion de transphonographie. Adaptée à la musique

1 Plusieurs reprises de la chanson «Summer Night City» ont été réalisées; il convient de mentionner entre autres celle du musicien funk Nils Langdren (Funky ABBA/ ACT Music, 2004) et celle du réalisateur EDM Offer Nissim (First Time/ Star 69 Records, 2005).

2 Cette reprise apparaît sur une version digipak de l'album Secret of the Runes (2001).

3 Dans cet article, le terme «transformation formelle» est emprunté à Gérard Genette, qui l'utilise pour désigner l'ensemble des modifications apportées à un texte (p. ex : traduction, versification). Adapté à l'analyse de la musique populaire, ce terme ne désigne donc pas uniquement la transformation de la structure d'une œuvre (comme l'emploi du terme laisse présager en musicologie), mais l'ensemble des transformations musicales pouvant être répertoriées. 
populaire par le musicologue Serge Lacasse, cette méthodologie est issue de la transtextualité, elle-même élaborée par le littéraire Gérard Genette. Ainsi, la transphonographie peut se définir par «tout ce qui met en relation, manifeste ou secrète, [un phonogramme] avec d'autres [phonogrammes] (Genette 1992, p. 7). Dans le cadre de cette analyse, je me concentrerai sur l'angle hyperphonographique, que j'expliquerai davantage dans l'introduction du premier chapitre.

J’emploierai également certains éléments propres à la narratologie, une méthodologie analysant cette fois la notion de récit, également employée par Genette et adaptée à la musique populaire par Lacasse. Enfin, je baserai mon analyse formelle sur la grille analytique de la musique populaire élaborée par Lacasse et comprenant : les paramètres abstraits (texte, forme, harmonie, mélodie) et les paramètres concrets, tels que ceux liés à la performance (p. ex. timbres, inflexions vocales) et à la technologie (p. ex. ajout de déphasage, saturation, réverbération, etc.) (Lacasse 2005).

\section{LES TRANSFORMATIONS FORMELLES DE «SUMMER Night CitY» : UN EXEMPLE DE TRANSPOSITION}

Dans l'analyse de la musique populaire, la notion de transphonographie s'avère particulièrement pertinente, puisqu'elle permet de rendre compte de relations existantes entre différents enregistrements de musique populaire ${ }^{4}$. En effet, une importante portion du répertoire populaire est bâtie sur des enregistrements antérieurs, qui sont réarrangés sous forme de remix, de parodies ou de travestissements (Lacasse 2008, p. 11). La transposition renvoie donc à cette notion de transphonographie, tout en relevant plus spécifiquement de la pratique de l'hyperphonographie, qui fait plus particulièrement référence à « toutes [les] relations unissant un [phonogramme] B (que j'appellerai un hypertexte) à un [phonogramme] $\mathrm{A}$ (que j'appellerai un hypotexte) sur lequel il se greffe d'une manière n'étant pas celle du commentaire» (Genette 1992, p. 13). Avant d'aller plus loin dans l'analyse, il est toutefois important de comprendre en quoi la transposition diffère de la parodie et du travestissement.

\section{La question du régime dans l'hyperphonographie}

Plus spécifiquement, un phonogramme peut être considéré comme une parodie lorsqu'il reprend de manière intégrale la musique de l'hypotexte, mais en modifiant de manière humoristique le contenu des paroles. Un travestissement, quant à lui, se définit comme un hypertexte reprenant intégralement le texte d'un hypotexte, mais en altérant le style de l'hypertexte, encore une fois de manière humoristique (Genette 1992, p. 35). Il serait donc juste de considérer la reprise de Therion comme un travestissement, puisqu'il s'agit bien d'une reprise intégrale des paroles en modifiant le style. Toutefois, le choix du terme transposition, au lieu du terme travestissement, repose la question du type de

4 De plus, la transphonographie comporte six grandes catégories : l'archiphonographie, l'hyperphonographie, l'interphonographie, la polyphonographie, la paraphonographie et la métaphonographie. Pour plus d'information, voir l'article de Lacasse (2008) sur le sujet. 
régime (la fonction de la musique) dans lequel évolue l'hypertexte. Ainsi, bien que le travestissement entraîne une modification du style, il découle historiquement du régime satirique, où l'intention est de tourner un texte en dérision. Toutefois, les transformations opérées par Therion ne s'insèrent pas dans un tel régime, mais appartiennent plutôt au régime sérieux. Ainsi, Genette emploie le terme transposition lorsqu'il fait référence aux transformations formelles et thématiques sérieuses pouvant survenir dans un hypertexte. De ce fait, la transposition est un genre hypertextuel (au même titre que le travestissement ou la parodie), qui modifie l'hypotexte par le biais de transformations formelles ou thématiques (Genette 1992, p. 43), mais dans un régime sérieux. Laissons d'abord de côté les transformations thématiques pour nous concentrer sur les transformations formelles, affectant le style et la forme.

En musique populaire, ces transformations formelles auront un impact sur les paramètres abstraits ou plus spécifiquement sur les éléments en lien avec le texte, la forme, l'harmonie et la mélodie. De plus, ces transformations peuvent également avoir une incidence sur les paramètres concrets, ceux affectant les timbres vocaux ou instrumentaux et les effets sonores présents dans les enregistrements (p. ex. réverbération, écho, saturation). Ainsi, dans la musique populaire, la transposition s'applique à une chanson dont les paramètres abstraits et concerts sont en partie conservés, tout en étant adaptés à un style musical différent, comme c'est le cas ici avec cette adaptation gothique metal symphonique de la musique disco. Dans ce premier chapitre, j'identifierai les paramètres abstraits et concrets se retrouvant dans la chanson "Summer Night City» de ABBA. Ce faisant, je serai ensuite en mesure d'établir les transformations formelles présentes dans la transposition de Therion.

\section{Les paramètres abstraits et concrets de «Summer Night City» de ABBA}

Écrite et composée par Björn Ulvaeus et Benny Andersson de ABBA, la chanson «Summer Night City» est lancée en $1978^{5}$ et devient rapidement un succès (Palm 2008, p. 384). Sur le plan musical, la chanson s'insère dans le courant de l'eurodisco, qui se développe en Europe dès la moitié des années 1970. Découlant du disco, l'eurodisco est souvent décrit comme une version plus «léchée» du disco américain. En effet, il se caractérise généralement par une ligne vocale simple, accompagnée d'une ligne de basse peu syncopée et par un synthétiseur reproduisant certains instruments de l'orchestre. De plus, le rythme en 4/4, accentuant chaque temps, est généré par une boîte à rythmes et demeure un des éléments les plus distinctifs de ce genre. Enfin, les thématiques traitées sont en lien avec les fêtes, la danse et la romance (Brackett 2012).

En outre, ces éléments sont également présents dans la chanson à l'étude. Ainsi, «Summer Night City» traite des sorties entre amis en ville lors des nuits d'été et des lointains souvenirs qui subsistent le matin suivant. Sur le plan des paramètres abstraits, cette chanson possède une forme simple comprenant deux couplets, un tremplin et un refrain, répétés deux fois.

5 ABBA. 1978. "Summer Night City», Summer Night City, Single, Polar Music International AB. 


\section{Tableau 1: Structure musicale de la chanson "Summer Night City" de ABBA}

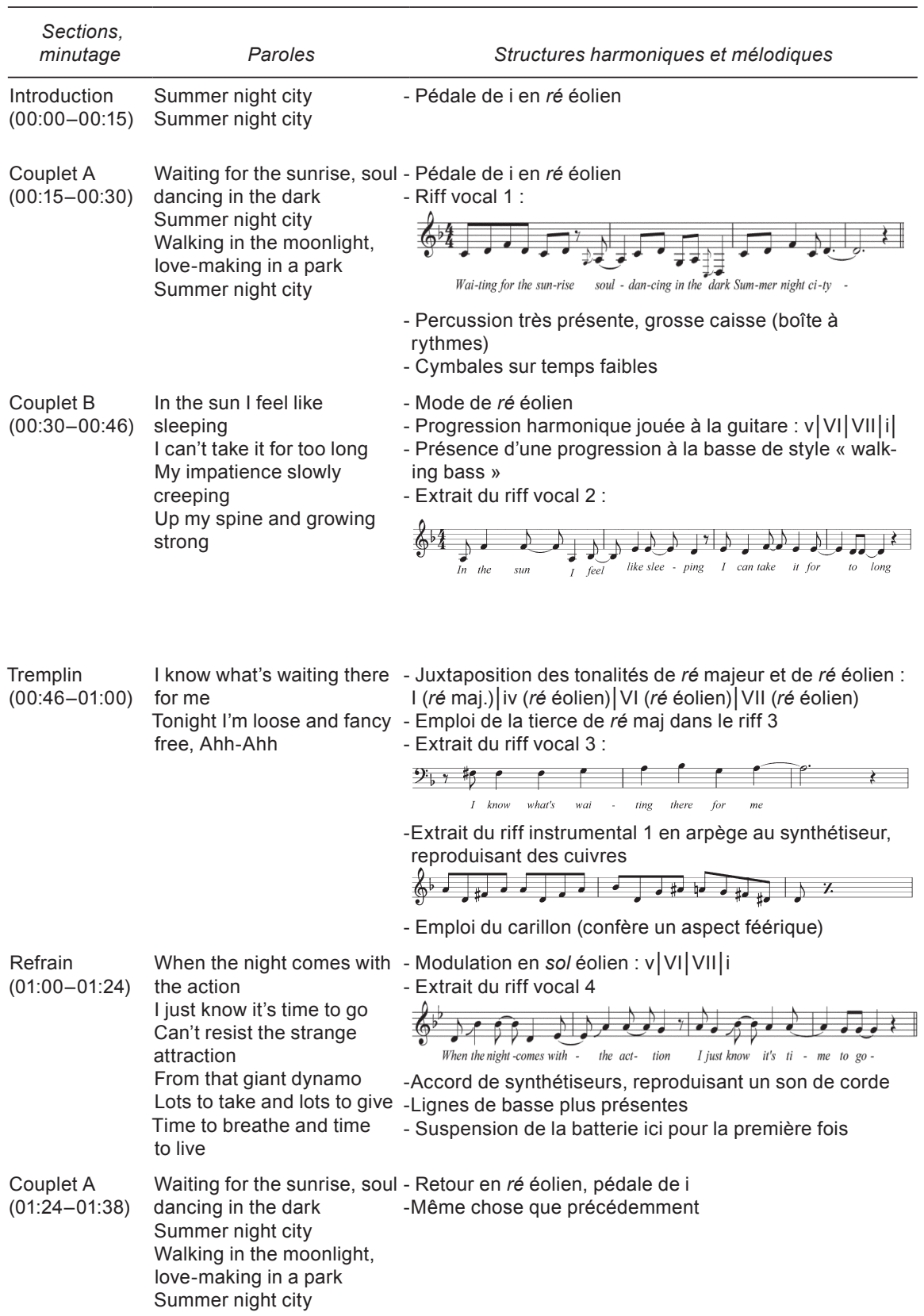




\section{Tableau 1 (continué)}

\begin{tabular}{|c|c|c|}
\hline $\begin{array}{l}\text { Sections, } \\
\text { minutage }\end{array}$ & Paroles & Structures harmoniques et mélodiques \\
\hline $\begin{array}{l}\text { Couplet B' } \\
(01: 38-01: 54)\end{array}$ & $\begin{array}{l}\text { It's elusive call it glitter } \\
\text { Somehow something turns } \\
\text { me on } \\
\text { Some folks only see the } \\
\text { litter } \\
\text { We don't miss them when } \\
\text { they're gone }\end{array}$ & Même chose que précédemment \\
\hline $\begin{array}{l}\text { Tremplin } \\
(01: 54-02: 09)\end{array}$ & $\begin{array}{l}\text { I love the feeling in the air } \\
\text { My kind of people } \\
\text { everywhere }\end{array}$ & Même chose que précédemment \\
\hline $\begin{array}{l}\text { Refrain } \\
(02: 09-02: 46)\end{array}$ & $\begin{array}{l}\text { When the night comes with } \\
\text { the action } \\
\text { I just know it's time to go } \\
\text { Can't resist the strange } \\
\text { attraction } \\
\text { From that giant dynamo } \\
\text { And tomorrow, when it's } \\
\text { dawning } \\
\text { And the first birds start to } \\
\text { sing } \\
\text { In the pale light of the } \\
\text { morning } \\
\text { Nothing's worth } \\
\text { remembering } \\
\text { It's a dream, it's out of } \\
\text { reach } \\
\text { Scattered driftwood on a } \\
\text { beach }\end{array}$ & Même chose que précédemment \\
\hline $\begin{array}{l}\text { Coda } \\
(02: 46-03: 47)\end{array}$ & $\begin{array}{l}\text { Waiting for the sunrise, soul } \\
\text { dancing in the dark } \\
\text { Summer night city } \\
\text { Walking in the moonlight, } \\
\text { love-making in a park } \\
\text { Summer night city } \\
\text { Waiting for the sunrise, soul } \\
\text { dancing in the dark } \\
\text { Summer night city } \\
\text { Walking in the moonlight, } \\
\text { love-making in a park } \\
\text { Summer night city } \\
\text { Waiting for the sunrise, soul } \\
\text { dancing in the dark } \\
\text { Summer night city } \\
\text { Walking in the moonlight, } \\
\text { love-making in a park } \\
\text { Summer night city }\end{array}$ & Retour en ré éolien, pédale de ré \\
\hline
\end{tabular}

De plus, les tonalités principales, en ré et sol éolien, sont présentées par le biais de patrons harmoniques assez simples. On retrouve également une alternance entre les tonalités de ré majeur et ré éolien dans les tremplins (voir schéma 1 en annexe). Plusieurs riffs vocaux peuvent aussi être entendus tout au long de la chanson, ainsi que différents riffs instrumentaux s'articulant sous forme d'ostinato. La chanson est construite sur un rythme en 4/4 qui est rapide (environ 132 à la noire) et stable, où chaque temps est accentué à la grosse caisse, tandis que les temps faibles sont appuyés par la charleston. 
Quant aux paramètres concrets, on remarque que les quatre voix entendues (deux hommes et deux femmes) sont de type populaire et qu'elles sont caractérisées par un timbre voilé et par quelques inflexions vocales ${ }^{6}$. L'instrumentation est également très standard et comprend plusieurs instruments reproduits au synthétiseur, dont des sons de cuivre (tremplins) et de cordes (refrains). Qui plus est, c'est la grosse caisse, exécutée par une boîte à rythmes, qui est à l'avant-plan et qui donne l'impulsion à la chanson. Il faut aussi noter l'utilisation d'un carillon lors des deux tremplins, conférant un aspect féérique à la musique. Enfin, cette chanson se veut joyeuse, légère et dégage un certain charme sensuel.

\section{La transtylisation métal de Therion}

C’est au cours des années 1990 que le metal symphonique se développe, en favorisant la fusion des sonorités de type symphonique propre à la musique classique avec celles plus lourdes et saturées caractérisant la musique metal. Ainsi, les groupes fondateurs de ce style, tels que Therion, Nightwish et Apocalyptica, ajoutent à leur musique metal des instruments de l'orchestre ou des synthétiseurs reproduisant ces instruments (Hein 2003, p. 51). Dans le cas plus spécifique de Therion7, des éléments propres à la musique gothique sont également présents ${ }^{8}$, grâce au rythme lent et lourd, aux voix plaintives, à l'ajout de différents effets sonores et aux modes mineurs employés, conférant un aspect mélancolique. Par ailleurs, sur le plan visuel, le groupe arbore une esthétique sombre (vêtement de type victorien, cheveux noirs, teint pâle), généralement associée au courant gothique (Gunn 1999, p. 37) ${ }^{9}$. Ainsi, le gothique metal symphonique désigne les groupes mettant de l'avant les sonorités agressives propres à la musique metal, auxquelles sont ajoutées des instruments classiques (ou des synthétiseurs les imitant), le tout évoluant dans une esthétique nostalgique et sombre propre à la musique gothique.

Dans la transposition de Therion, on peut noter quelques changements sur le plan des paramètres abstraits et concrets, qui s'insèrent dans l'esthétique

6 Par exemple, on peut entendre plusieurs portamentos, qui sont identifiés dans les différents riffs vocaux du tableau précédent.

7 Fondé par Christopher Johnsson en 1987, Therion a 15 albums à son actif et est reconnu pour avoir popularisé l'utilisation d'instruments et de voix classiques dans le contexte metal. Les thématiques explorées par le groupe portent principalement sur le mysticisme, l'ésotérisme et la mythologie nordique.

8 Voir à ce propos les albums Theli (1996) et Gothic Kabbalah (2007)

9 Communément, le terme "gothique» réfère à deux mouvements artistiques; d'abord, il est employé pour désigner l'architecture de type gothique présente en Europe (ca. $15^{\mathrm{e}}$ siècle) et s'applique aussi au courant littéraire de la fiction gothique (ca. fin du $18^{\mathrm{e}}$ siècle) en provenance de l'Angleterre. Plus spécifiquement, la fiction gothique, où l'action se déroule souvent dans différents lieux à l'architecture gothique (p.ex. manoirs, cimetières, églises), met l'accent sur les thèmes du mystère, du diable et de la noirceur humaine. Au début des années 1980, l'appellation "gothique» est reprise par les groupes de post-punk britannique, tels que Bauhaus, Joy Division et the Damned. Malgré le peu d'indices permettant d'identifier la filiation entre l'architecture, la littérature et la musique gothique, des constantes esthétiques (particulièrement avec la fiction gothique) sont néanmoins conservées par ces groupes : les thèmes lyriques de la mort et de la destruction sont souvent abordés tout en étant intimement liés avec un romantisme morbide (Gunn 1999, p. 37). 
musicale privilégiée par le metal symphonique. Plus spécifiquement, ces modifications peuvent être décrites comme des substitutions, puisque tous les éléments (texte, forme, harmonie, mélodie) sont présents, mais sont adaptés au langage musical du groupe. Tout d'abord, la forme est conservée et deux légères modifications textuelles peuvent être identifiées : une excision et une substitution. Ainsi, dans le couplet $\mathrm{A}$, les mots « dark» et «park» ont été retirés de l'hypertexte. De plus, dans la coda, les paroles du couplet A de l'hypotexte sont remplacées par les paroles des deux tremplins. Toutefois, malgré ces deux modifications, les paroles sont reprises intégralement.

\section{Tableau 2: Transformations de la chanson "Summer Night City" par Therion}

\begin{tabular}{|c|c|c|}
\hline $\begin{array}{l}\text { Sections } \\
\text { minutage }\end{array}$ & $\begin{array}{l}\text { Paroles et structures } \\
\text { harmoniques }\end{array}$ & Transformations \\
\hline $\begin{array}{l}\text { Intro } \\
(00: 00-00: 57)\end{array}$ & $\begin{array}{l}\text { i-Il (alternance entre les } \\
\text { deux degrés) }\end{array}$ & $\begin{array}{l}\text { - Substitution mélodique : remplacement du mode de ré } \\
\text { éolien par le } 5^{\mathrm{e}} \text { mode mineur harmonique, aussi appelé } \\
\text { le mode oriental. } \\
\text { - Extrait du riff instrumental } 1 \text { joué aux cordes }\end{array}$ \\
\hline
\end{tabular}

Couplet A Waiting for the sunrise, (00:57-01:16) soul dancing in the Summer night city Walking in the moonlight, love-making in the Summer night city

Couplet B (01:16-01:34)

Tremplin (01:34-01:51)
In the sun I feel like sleeping I can't take it for too long My impatience slowly creeping

Up my spine and growing strong

know what's waiting there for me Tonight I'm loose and fancy free, Ahh-Ahh
- Substitution harmonique : remplacement de la pédale de ré éolien par une nouvelle progression toujours en ré phrygien : i| III|II

- La mélodie est toutefois en ré éolien

- Excision des mots "dark" et "park"

- Guitare rythmique saturée et guitare claire arpégée

- Mélodie du riff vocal 1 est la même que l'hypotexte

- Voix classiques solennelles

- Conservation du mode de ré éolien

- Progression légèrement modifiée pour : i|VI|VII|i

- Voix masculine au timbre légèrement strident et agressif, accent sur certaines voyelles

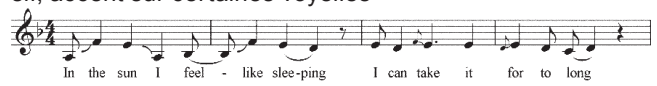

- Contour mélodique du riff vocal 2 est sensiblement le même que l'hypotexte

- Conservation des tonalités de ré majeur et de ré éolien, mais progression harmonique légèrement modifiée :

I|VII|VI|iv|I|VII|VI|V6

- Extrait du riff vocal 3, transposition à une voix de mezzo-soprano

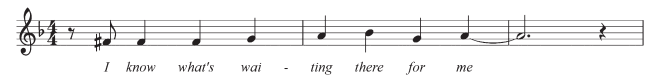

- Extrait du riff instrumental 2 joué au hautbois

- Transformation du contour mélodique et du registre. Débute une tierce mineure plus bas.

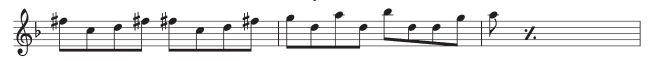




\section{Tableau 2 continué}

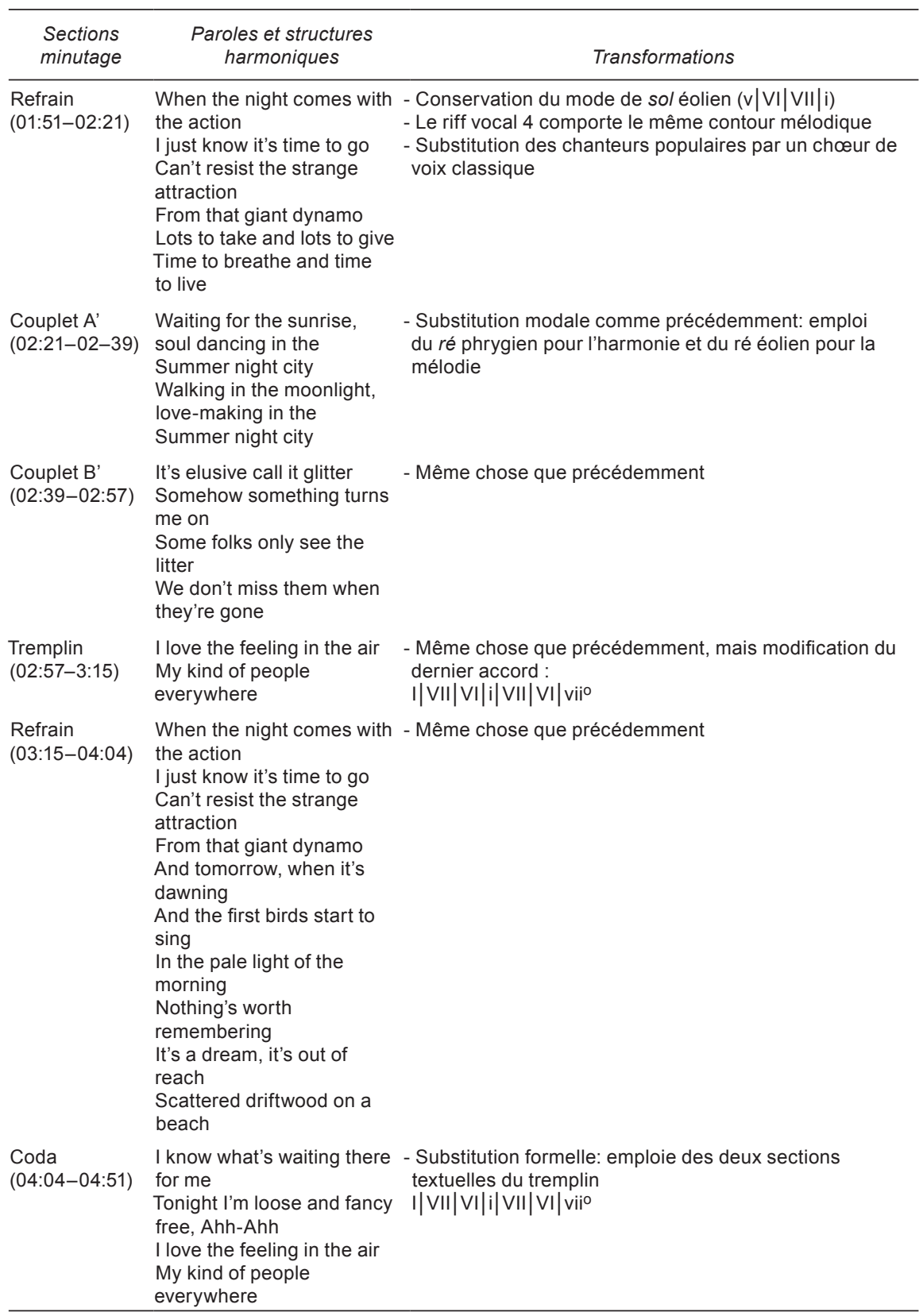

En outre, on peut voir des changements sur les plans de l'harmonie et de la mélodie. Ainsi, dans l'introduction, le mode de ré éolien de l'hypotexte n'est pas conservé, ce qui engendre aussi une transformation mélodique. En effet, à cet endroit, le riff instrumental joué aux cordes est construit sur le $5^{\mathrm{e}}$ mode 
mineur harmonique, aussi appelé gamme orientale; la mélodie comporte à la fois une seconde abaissée et un troisième degré majeur (voir schéma 1 en annexe). Les couplets A et A' comprennent également une nouvelle progression harmonique, où la pédale de i en ré éolien est modifiée pour la progression i|III|II en ré phrygien. Toutefois, la mélodie vocale de l'hypotexte en ré éolien est conservée. Une autre transformation, mélodique cette fois, est présente dans le tremplin, puisque le riff instrumental interprété au hautbois débute une tierce mineure plus bas que dans la version d'origine et ne comporte pas exactement le même contour mélodique (voir schéma 1 en annexe). Cependant, ces modifications n'ont pas d'impact majeur sur la structure, puisque les modes et les accords privilégiés demeurent sensiblement les mêmes que dans l'hypotexte. De plus, l'utilisation du mode phrygien dans cette transposition de Therion est une caractéristique propre à la musique metal, puisqu' il confère une sonorité plus sombre à la chanson ${ }^{10}$. Enfin, l'autre principale transformation est rythmique, puisque la chanson est jouée dans un tempo plus modéré (environ 108 à la noire) que l'originale (environ 132 à la noire).

Toutefois, les transformations formelles sont davantage visibles au niveau des paramètres concrets. En effet, il convient de parler avant tout de l'instrumentation, qui reflète en tout point le langage musical du groupe. Par conséquent, l'instrumentation plus standard de ABBA est étendue grâce à l'incorporation de quelques instruments de l'orchestre (violon, alto, violoncelle, hautbois), auxquels s'ajoutent les instruments amplifiés. Ainsi, le riff entendu dans l'introduction est maintenant exécuté par les cordes et celui du tremplin par le hautbois, en remplacement du synthétiseur. De plus, cette nouvelle version met à l'avant-plan les sonorités de guitares électriques, l'une saturée et l'autre claire. En outre, l'interprétation de la batterie, où cette fois le beatbox n'est plus employé, se caractérise par une accentuation des temps faibles et par un usage plus restreint de la charleston.

Enfin, en remplacement des quatre voix de type populaire présentées dans la version d'origine, Therion en emploiera plutôt cinq. De plus, la majorité des voix employées sont de type classique, à l'exception d'une voix masculine, de type populaire. D'ailleurs, cette voix se démarque grâce aux nombreuses inflexions qui ornementent la ligne vocale. Le timbre légèrement agressif et strident (souvent employé dans la metal gothique), ainsi que la liberté d'interprétation dans la ligne vocale (p.ex. certaines voyelles seront volontairement allongées) semble lui conférer une certaine arrogance n'étant pas perceptible dans l'hypotexte. D'ailleurs, cette alternance entre les timbres classiques et les timbres agressifs demeure très employée dans la musique metal symphonique (Hein 2003, p. 51). Ainsi, la chanson joyeuse et légère de ABBA s'alourdit et s'assombrit, grâce aux instruments amplifiés, au rythme plus modéré et à la voix masculine de type populaire. En outre, le charme sensuel qui s'en dégageait à

10 Le mode phrygien est très employé dans la musique metal en raison de la seconde abaissée qui le caractérise et qui introduit une dissonance, conférant ainsi une sonorité plus sombre à la musique (Cope 2010, p. 53-54). 
l'origine est remplacé par une atmosphère solennelle, en raison de l'utilisation des instruments classiques et des voix lyriques.

En plus des transformations formelles évidentes qu'entraîne cette transposition, le vidéoclip de cette chanson conçu par Therion, présente des transformations thématiques encore plus importantes, qui seront analysées dans la deuxième portion de cette analyse.

\section{LES TRANSFORMATIONS THÉMATIQUES DU VIDÉOCLIP «SUMMER Night City » De Therion}

Lorsqu'on compare la version du vidéoclip «Summer Night City» réalisée par Therion avec celle de ABBA, on remarque qu'en plus des nombreuses transformations formelles, de nombreuses transformations thématiques sont présentes. Selon Genette, les transformations thématiques pouvant survenir dans un hypertexte se regroupent en trois catégories : les transformations diégétiques, qui touchent l'univers spatio-temporel, les transformations pragmatiques, qui influencent la conduite de l'action et enfin les transformations sémantiques, qui elles, affectent la signification de l'hypertexte. Or, en plus des transformations évoquées, Therion propose une diégèse et un récit s'éloignant considérablement du l'hypotexte de ABBA. Comme aucune catégorie de Genette ne semble s'appliquer à ce type précis de transformation, qui se rapporte tant au récit qu'à la diégèse, je qualifierai ce type de transformation de diégético-narrative. Enfin, il sera démontré que le récit proposé par Therion se rapproche de l'idéologie propre au metal.

Dans cette deuxième portion de l'analyse, j'identifierai les différentes transformations thématiques, tant diégético-narratives, pragmatiques que sémantiques, décelables dans le vidéoclip «Summer Night City» réalisé par Therion. Avant tout, une première section de l'analyse traitera de la notion de récit, que j’appliquerai ensuite pour analyser le vidéoclip original de ABBA.

\section{La notion de récit en narratologie et son application à la musique populaire}

La narratologie est une méthodologie employée en étude littéraire qui porte sur la notion de récit. Plus spécifiquement, le terme récit fait référence à la manière dont les éléments de l'action, donc l'histoire, sont rapportés par un narrateur. En plus de prendre pied dans un univers diégétique spécifique, le récit se déploie dans le temps. Ainsi, les éléments de l'histoire sont présentés dans un certain ordre et ils occupent une durée variable selon l'importance qui leur est accordée. Le récit est aussi construit d'après le rôle que le narrateur occupera dans l'histoire. En outre, le narrateur peut être impliqué activement dans l'histoire qu'il raconte, ou bien seulement rapporter des événements auxquels il n’a pas pris part. Enfin, le récit sera également influencé par le type de discours que le narrateur emploiera pour raconter l'histoire. Par exemple, le récit peut être rapporté (narrativisé) par le narrateur ou bien retransmis par les personnages, grâce au dialogue (Genette 2007, p. 19).

Bien que la chanson populaire ne puisse être considérée selon les mêmes critères qu'une œuvre littéraire, Lacasse spécifie qu'elle n'en demeure pas moins 
un récit. Ainsi, tout comme dans le cas de la forme littéraire ou du théâtre, elle met en scène un narrateur et des personnages qui s'expriment face à une situation quelconque (Lacasse 2006, p. 13). Pourtant, comme l'explique Lacasse, la chanson s'apparente plutôt au cinéma, en offrant une mise en scène "phonographique». Plus spécifiquement, dans la chanson populaire, la mise en scène phonographique des différents paramètres sonores (abstraits et concrets) participe à l'élaboration et à la compréhension de ce récit (Idem). Dans ce contexte, le récit et sa diégèse, en plus d'être construits à partir du texte, sont mis en scène grâce aux éléments musicaux employés. Comme il sera démontré, l'ensemble des concepts propres au récit littéraire, tels que le temps, le mode et la voix, peut également s'appliquer à la musique populaire.

\section{La diégèse, l'histoire et le récit de «Summer Night City» de ABBA}

Tout d'abord, dans l'hypotexte à l'étude, il est important de spécifier que la diégèse, l'histoire et le récit sont à peine esquissés, bien qu'ils soient renforcés par la mise en scène phonographique des éléments musicaux et visuels. Ainsi, lorsque l'on examine le texte de la chanson de ABBA, on remarque que l'histoire et la diégèse demeurent peu précises. En effet, la chanson évoque grosso modo les soirées d'été entre amis passées à danser au rythme de la musique et connote les souvenirs qui subsistent le matin venu. Toujours dans le texte, les lieux de l'action sont seulement esquissés : le récit semble se dérouler dans un contexte urbain, à la fois durant le jour et la nuit (voir schéma 2 en annexe). Toutefois, la musique et le vidéoclip de ABBA donne davantage de substance à l'histoire et à la diégèse : on réalise que l'histoire se déroule principalement la nuit dans une ville, et plus spécifiquement dans une discothèque. En effet, les mouvements de danse joints aux rythmes répétitifs de la grosse caisse et aux effets d'éclairage illustrent clairement le lieu, tout en insufflant une certaine frénésie à l'histoire. De plus, les timbres spécifiques des instruments et les éléments du décor (habillement, coiffures, automobiles) nous ramènent dans l'univers des années 1970.

Encore une fois, lorsqu'on examine les paroles, les éléments propres au temps du récit demeurent peu détaillés. Ainsi, l'ordre des événements de l'histoire suggérée par le texte semble être le suivant : l'attente du début de la soirée (couplet B et tremplin 1), la frénésie de la danse (refrain 1, tremplin 2, couplets A et A', et couplet B') et enfin les souvenirs de la soirée le lendemain (refrain 2). Toutefois, le déroulement spécifique de ces événements demeure peu précis et est construit sous forme de prolepses et d'analepses; chacun des segments temporels fait allusion à des événements à venir (p. ex. l'attente du début de la soirée) ou s'attarde sur des événements passés (p.ex. le souvenir de la soirée le lendemain tout vraisemblablement) (Genette 2007, p. 59). L'ordre temporel sera légèrement renforcé par le vidéoclip : on peut voir les personnages se rendre à la soirée, danser et ensuite se promener dans la ville, le jour. Toutefois, comme il a été démontré, la trame événementielle est peu supportée par le texte ou les images, et demeure seulement suggérée. Quant à la durée de l'histoire, elle est ambiguë, car ce récit semble faire référence tant à une soirée spécifique qu’à 
toutes les soirées de ce type. Par ailleurs, ni le vidéoclip ni la musique ne renforcent cette notion de durée.

Toujours dans le texte, peu d'indices sont fournis quant au type de narrateurs ou aux personnages jouant un rôle dans l'histoire. En effet, en plus de s'appliquer aux deux genres (masculin et féminin), le texte semble ne présenter qu'un seul narrateur. Toutefois, le narrateur peut être considéré comme étant homodiégétique, puisqu'il n'est pas seulement un spectateur, mais participe à l'action de l'histoire qu'il raconte. Pourtant, tant les images du vidéoclip que les timbres vocaux masculins et féminins nous informent plutôt que la chanson s'articule d'après les propos de plusieurs personnages, mais sans déterminer précisément leur rôle (personnages principaux ou secondaires). Enfin lorsqu'on examine le type de discours employé dans les paroles, on réalise qu'il ne comporte aucun dialogue, mais que les interventions semblent être construites sous forme de discours intérieurs prononcés tout haut, où les personnages partagent leur état d'âme ${ }^{11}$. En outre, dans le vidéoclip, les interventions des personnages sont rapportées directement à l'auditeur, renforçant la thèse du discours intérieur prononcé à voix haute. Voyons maintenant comment Therion s'approprie ces éléments en les adaptant à son esthétique spécifique.

\section{Les transformations diégético-narratives et pragmatiques du vidéoclip "Summer Night City» de Therion}

Grâce à la diégèse et au récit peu développés du texte de $\mathrm{ABBA}$, Therion a été en mesure d'utiliser le squelette de l'histoire de ABBA pour procéder ensuite à une série de transformations diégétiques et narratives, qui auront un impact significatif sur le temps, la voix et le mode du récit. De plus, ces transpositions diégético-narratives influenceront le déroulement pragmatique de l'action, que j'aborderai plus loin.

Dans le vidéoclip de Therion, plusieurs indices permettent d'établir que la diégèse du récit prend place dans les années 1930, dans un manoir en campagne. En effet, au tout début du vidéoclip, avant même que la chanson ne commence, on peut voir une locomotive à vapeur démarrant lentement du quai d'embarquement (oo:00-00:22). D’ailleurs, cette mise en contexte peut même être vue comme un élément permettant à l'auditeur de pénétrer dans cette diégèse très distincte. En outre, les personnages portent des vêtements rappelant cette époque et se déplacent dans une automobile datant des années 1930. Cette transformation diégétique engendre donc ce qu'il convient d'appeler une translation temporelle, puisque la diégèse à laquelle Therion fait référence diffère de celle mise en place dans l'hypotexte. Un phénomène de distanciation ${ }^{12}$

11 Bien que la voix soit constamment audible dans une chanson, le discours d'un personnage peut correspondre soit à un monologue intérieur (voix non prononcée) soit à un discours énoncé à voix haute (prononcée) décrivant les états d'âme du personnage (Lacasse 2006, p. 20).

12 Dans son ouvrage sur la transtextualité, Genette ne mentionne pas le phénomène de distanciation employé ici. Toutefois, il fait référence à l'effet de proximisation lorsque l'histoire de l'hypertexte se déroule dans un cadre diégétique se rapprochant de la réalité du lecteur (Genette 1992, p. 431). Il est donc logique d'employer le terme distanciation pour faire référence au phénomène inverse. 
peut aussi être noté, étant donné que cet hypertexte propose une diégèse plus éloignée dans le temps que celle d'origine.

Sur le plan du temps du récit, cette nouvelle version comporte une chronologie beaucoup plus détaillée. En effet, le vidéoclip est constitué d'éléments d'action très différents, mais tout en présentant en filigrane l'histoire de l'hypotexte. Par exemple, le vidéoclip débute (introduction) par le voyage d'une femme dans un train, où celle-ci, à partir du tremplin 1, évoque la soirée à laquelle elle participera sous peu : «I know what's waiting there for me...» (01:34). Alors qu'elle est raccompagnée en voiture par son prétendant vers le manoir (couplet B'), ce dernier lui explique à quel point la nuit de fête qui les attend sera agréable : «It's elusive, call it glitter, somehow someting turns me on...» (o2:39). Lors de l'arrivée des deux protagonistes au manoir (refrain 2), plusieurs convives célèbrent en s'exhortant à oublier dès le lendemain les folies commises au cours de leur soirée : «In the pale light of the morning nothing's worth remembering» (03:43). Il est donc évident que cet ordre des événements, malgré qu'il soit transposé à une diégèse différente, est directement tiré de l'hypotexte. La trame événementielle est d'ailleurs renforcée grâce aux différentes interventions des personnages, ayant un timbre de voix spécifique, qui accompagne les segments temporels de la chanson. De plus, la durée du récit est beaucoup plus claire : il s'agit d'un récit se déroulant sur une seule journée, puisqu'il fait référence à un événement précis. Toutefois, la durée du récit parait ici beaucoup plus longue, puisque le rythme pesant et saturé de la guitare contribue à alourdir la vitesse d'enchaînement des éléments.

Sur le plan de la voix du récit, Therion crée des personnages tangibles, qui interagissent entre eux tout au long du vidéoclip. Ainsi, on note deux personnages principaux (la jeune femme se rendant à la fête et son prétendant), ainsi que des personnages secondaires (les invités au manoir et les majordomes). Une transexuation ${ }^{13}$ est également visible, puisque les interventions hommesfemmes de la version d'origine ne sont pas conservées, ce qui confère une thématique complètement différente à l'hypertexte (Genette 1992, p. 423). Ainsi, chacune des sections du texte est réservée à l'intervention d'un des personnages principaux ou secondaires, ce qui permet une meilleure compréhension de ce nouveau récit. Par exemple, les majordomes interviennent dans les couplets A et $A^{\prime}$; la jeune femme prend la parole dans les deux tremplins; le prétendant interviendra dans les couplets B et B', et les invités à la fête, s'exprimeront lors des refrains. La coda se démarquera par l'intervention des deux personnages principaux, ainsi que des invités (voir schéma 2 en annexe).

Comme Genette le spécifie, cette modification dans la construction des interventions des personnages constitue une transformation hétérodiégétique, puisque tant le cadre de l'action que les personnages sont altérés dans cet hypertexte (Genette 1992, p. 422). Enfin, le type de discours varie tout au long du vidéoclip passant du dialogue au discours intérieur, ce qui permet de mieux comprendre le déroulement de l'histoire telle que racontée par Therion. Ainsi,

13 Une transexuation survient lorsqu'un des personnages de l'hypotexte change de genre sexuel (un homme devient une femme et vice-versa) dans l'hypertexte (Genette 1992, p. 423). 
dans le couplet $\mathrm{A}$, ce sont d'abord les majordomes, reconduisant le prétendant à la gare, qui évoquent la frénésie des soirées d'été sous forme de discours intérieur : "Waiting for the moonlight, soul dancing in the...» (oo:57). Puis, dans le couplet $\mathrm{B}$, le prétendant confie à ses majordomes la difficulté qu'il éprouve à devoir attendre la tombée de la nuit : «In the sun I feel like sleeping, I can’t take it for too long" (01:16). La focalisation se déplace ensuite sur la jeune femme dans le train (tremplin), qui, par-devers elle, exprime l'excitation qu'elle ressent à l'idée de la soirée qui l'attend : «I know what's waiting there for me» (01:34). $\mathrm{Au}$ cours du voyage en automobile vers le manoir (couplet B'), un dialogue est également perceptible entre les deux personnages principaux, lorsque le prétendant évoque la frénésie qui s'empare de lui lors des fêtes («It’s elusive call it glitter, somehow something turns me on») (02:39). Puis, à son arrivée au manoir (tremplin), la jeune femme, accueillie par les invités, leur explique combien elle se sent acceptée parmi eux : «I love the feeling in the air; My kind of people everywhere» (03:15) (voir schéma 2 en annexe).

Évidemment, ces transformations diégético-narratives entraînent aussi des transformations pragmatiques ${ }^{14}$, puisque l'action est déplacée des années 1970 aux années 1930. Certains éléments sont donc forcément transposés : la fête au manoir en remplacement de la discothèque, l'ajout de la locomotive, la présence d'une automobile d'époque, les vêtements anciens et enfin l'attitude des personnages, qui agissent de manière plus distinguée et moins frivole. Pourtant, le changement le plus évident demeure en lien avec le traitement visuel. Ainsi, les lumières scintillantes caractérisant le vidéoclip de ABBA sont ici remplacées par des images aux teintes sépia, accentuant l'aspect vieillot.

Toutefois, des transpositions pragmatiques de cet ordre peuvent entraîner une certaine étrangeté, comme dans les couplets A et A'. En effet, à cet endroit, les majordomes chantent le texte suivant : "Waiting for the moonlight, love making in the [park] », conférant un aspect grotesque à cette section. En effet, il est surprenant d'entendre une telle ligne de texte de la bouche de majordomes, dont l'attitude solennelle et la voix classique ne collent pas du tout avec les propos tenus.

\section{Les transformations sémantiques du vidéoclip et de la chanson «Summer Night City" de Therion}

Le dernier type de transformations thématiques, souvent engendré par des transpositions diégétiques et pragmatiques, se nomme transformation sémantique et affectera la signification de l'hypertexte (Genette 1992, p. 457). Ainsi, cette modification du sens de l'hypertexte se manifeste par une transmotivation ou une transvalorisation. Plus spécifiquement, par transmotivation Genette fait allusion à la substitution du motif original (soit le but ou l'objectif) de l'hypotexte par un nouveau (Genette 1992, p. 458). La transvalorisation, quant à elle, fait référence à «toute l'opération d'ordre axiologique, portant sur la

14 Des transformations pragmatiques surviennent lorsque l'histoire du l'hypertexte ne se déroule plus à la même époque que celle de l'hypotexte nécessitant alors certains ajustements dans la trame de l'action (Genette 1992, p. 442). 
valeur explicitement ou implicitement attribuée à une action» ou dans ce casci, à la valeur qui sera conférée à un style de musique (Genette 1992, p. 483). Dans cette dernière portion de l'analyse, je m'intéresserai donc aux transmotivations et aux transvalorisations d'ordre sémantique qui sont présentes tant dans le vidéoclip que dans l'enregistrement sonore de "Summer Night City» réalisés par Therion.

Tout d'abord, le vidéoclip de Therion comporte une importante transmotivation, puisque le motif de l'histoire est complètement transformé. Ainsi, le vidéoclip de $\mathrm{ABBA}$, dont le motif principal vise à recréer la frénésie des sorties entre amis, n'est plus le même dans la version de Therion. En effet, si dans l'hypotexte la sortie à la discothèque est l'élément principal du récit, dans cette nouvelle version, la réception au manoir devient un élément moins important. De plus, dans ce nouveau récit, l'histoire d'amour entre les deux personnages principaux prend beaucoup d'espace. Non seulement cette nouvelle motivation devient un élément d'action très important, mais elle ajoute une dimension romantique au récit, qui n'était pas présente dans l'hypotexte. D'ailleurs, par le biais du vidéoclip, Therion accentue cette atmosphère romantique et sombre propre à l'esthétique gothique grâce à la musique lourde et au timbre légèrement agressif du prétendant.

Une autre transformation sémantique est également présente : il s'agit d'une transvalorisation, tant sonore que visuelle. Tout d'abord, cette transposition de "Summer Night City» de ABBA par Therion peut être considérée comme une revalorisation à saveur metal de la musique de ABBA. Malgré que cette reprise d'une chanson disco par un groupe gothique metal symphonique puisse paraitre inusitée, Therion (étant originaire de Suède tout comme ABBA), reconnaît l'importance de ABBA dans l'histoire de la musique suédoise (Ronnie 2010). En adaptant cette chanson au langage metal symphonique, Therion exécute une véritable transvalorisation de la musique disco. Il permet donc aux amateurs de metal d'apprécier une chanson, qui autrement ne les intéresserait pas forcément, et ce, grâce à la substitution du vocabulaire disco par le vocabulaire gothique metal symphonique (Applebaum 2004).

Une autre transvalorisation, mais d'ordre visuel, est aussi à noter et se rapporte à l'utilisation de la diégèse «ancienne» caractérisant le vidéoclip de Therion. Loin d'être un élément anodin, cet attrait pour une époque révolue est extrêmement fréquent dans la musique metal. D’ailleurs, pour l'historien metal Nicolas Bénard, cette utilisation d'un cadre ancien doit être comprise comme une idéalisation des valeurs et du mode de vie du passé et comme une critique et même un rejet de la société de consommation actuelle (Bénard 2009, p. 68$)^{15}$. Ainsi, la transposition du cadre diégétique des années 1970 à celui des années 1930 pourrait avoir la même fonction, soit de critiquer la superficialité de la société et de la musique actuelle, et par extension celle des années 1970.

15 Par exemple l'emploi, par de nombreux groupes de sujets en lien avec le paganisme et la mythologie nordique (p. ex. Eluveitie, Satyricon, Ensiferum) peut être compris comme une critique face à l'uniformisation des cultures sur l'échelle planétaire (Bénard 2009, p. 68). 
Pour conclure, cette analyse a démontré que la transphonographie, et plus particulièrement l'hyperphonographie, est un outil très efficace pour mieux décrire et comprendre les transformations formelles et thématiques à l'œuvre dans la chanson «Summer Night City» de ABBA et reprise par le groupe metal symphonique Therion. Ainsi, Therion exécute une véritable adaptation de cette chanson disco en lui conférant une esthétique metal symphonique. De ce fait, les transformations appliquées à l'hypertexte sont d'abord visibles au niveau des paramètres abstraits et concrets : Therion amplifie la sonorité phrygienne (très employée dans la musique metal), ajoute des voix et des instruments classiques, qu'il joindra au son lourd et saturé de la guitare électrique.

Toutefois, lorsque l'on compare le vidéoclip de la chanson «Summer Night City» exécuté par Therion avec le vidéoclip original de ABBA, on remarque que de nombreuses transformations thématiques sont aussi présentes. Ainsi, plusieurs modifications sur les plans diégétique, narratif, pragmatique et sémantique sont à l'œuvre dans la transposition de la chanson par Therion. Plus spécifiquement, dans l'adaptation vidéoclip, Therion, en se basant sur l'histoire générale de la chanson de ABBA, brode un cadre diégétique et une trame narrative complètement différents. Ces modifications auront également un impact sur la signification de l'hypertexte, grâce à la substitution du motif original de l'histoire par un nouveau, et grâce à la valorisation de la musique de ABBA, mais adaptée à l'esthétique gothique metal symphonique. En somme, cette adaptation de Therion, où l'esthétique visuelle et musicale est transposée au vocabulaire metal, demeure un bel exemple du décalage idéologique que vivent les amateurs de metal par rapport à la société actuelle.

\section{RÉFÉRENCES}

Applebaum, Maor. 2004. Interview with : Christopher Johnsson from Therion, Alternative-Zine, 3 mai $2004<\mathrm{http}$ ///www.alternative-zine.com/interviews/ en/6> (consulté le 6 octobre 2011).

Bénard, Nicolas. 2009. «Les mythologies Hard Rock et Métal : bricolage identitaire ou récit original», Sociétés, 2009, $\mathrm{n}^{\mathrm{0}}$ 104, 65-72.

Cope, Andrew L. 2010. Black Sabbath and the Rise of Heavy Metal Music. Farnham, Surrey; Burlington, Vt. : Ashgate.

Genette, Gérard. 2007. Discours du récit. Paris : Seuil.

—_. 1992. Palimpsestes : La littérature au second degré. Paris : Seuil.

Gunn, Joshua. 1999. "Gothic Music and the inevitability of genre». Popular Music and Society 23, $\mathrm{n}^{\mathrm{o}} 1: 3$ 31-50. $^{2}$

Hein, Fabien. 2003. Hard rock, heavy metal, metal: Histoire, cultures et pratiquants. Nantes: M. Séteun; Paris: IRMA Éditions.

Lacasse, Serge. 2005. «La musique comme discours phonographique : Fondement d'une démarche d'analyse». Musicologies, $\mathrm{n}^{\circ} 2: 23-39$.

_. 2006. «Stratégies narratives dans "Stan" d'Eminem : le rôle de la voix et de la technologie dans l'articulation du récit phonographique». Protée 34, $\mathrm{n}^{\mathrm{os}} 2-3: 11-26$.

. 2008. «La musique pop incestueuse : une introduction à la transphonographie». Circuit: musiques contemporaines $18, \mathrm{n}^{\circ} 2: 11-26$. 
Palm, Carl Magnus. 2008. Bright Lights Dark Shadows: The Real Story of Abba. London :Omnibus Press.

Ronnie. 2010. «Entretien avec Thomas Vikström (chant)». Les Immortels,- le 09 septembre $2010<\mathrm{http} / / / \mathrm{www} \cdot$ leseternels.net/interviews.aspx?id=346> (consulté le 12 décembre 2012).

\title{
ENREGISTREMENTS
}

Langdren, Nils. 2004. «Summer Night City», Funky ABBA, ACT Music. Nissim, Offer. 2005. «Summer Night City», First Time, Star 69 Records. Therion. 1996. «Theli», Nuclear Blast.

Therion. 2001. "Summer Night City», Secret of the Runes, Nuclear Blast. Therion. 2009. "Gothic Kabbalah», Nuclear Blast.

ABBA. 1978. «Summer Night City», Summer Night City, Single, Polar Music.

\section{RÉSUMÉ}

Une des caractéristiques fondamentales de la musique populaire demeure la réutilisation constante du matériel musical en provenance d'une multitude de styles et de courants (remix, adaptation, échantillonnage, etc.). Cette pratique nommée la transphonographie provient de l'intertextualité, un concept élaboré par le littéraire Gérard Genette et adapté à la musique populaire par le musicologue Serge Lacasse. Dans ce texte, l'auteur emploiera la transphonographie pour relever les différentes transformations musicales et thématiques survenant dans la chanson "Summer Night City" de ABBA, lorsque celle-ci est reprise par le groupe de gothique metal symphonique Therion. En plus des éléments musicaux propres à la musique d'ABBA modifiés pour correspondre à l'esthétique de Therion, ces transformations induisent aussi un changement dans la signification même de la chanson.

\begin{abstract}
One of the main characteristics of popular music is still the reusing of musical material borrowed from a multitude of styles and trends (remix, adaptation, sampling, etc.). This practice is called transphonography, and stems from intertextuality, a concept developed by Gérard Genette, a literary scholar, and adapted to music by musicologist Serge Lacasse. In this article, the author references transphonography to study the various musical and thematic transformations in the gothic metal symphonic group Therion's reworking of ABBA's song "Summer Night City". Along the transformations of musical elements in order to adapt ABBA's music to Therion's aesthetics, some transformations induce a shift in the very significance of the song.
\end{abstract}

